\title{
Research
}

\section{The acceptability to patients and professionals of remote blood pressure monitoring using mobile phones}

\author{
Yvonne Bostock ${ }^{1}$, Janet Hanley ${ }^{2}$, Douglas McGown ${ }^{3}$, Hilary Pinnock ${ }^{4}$, Paul Padfield ${ }^{5}$ and \\ Brian McKinstry ${ }^{4}$ \\ ${ }^{1}$ Freelance researcher, Edinburgh, UK \\ ${ }^{2}$ Centre for Integrated Health Research, Edinburgh Napier University, Edinburgh, UK \\ ${ }^{3}$ Broxburn Health Centre, Broxburn, West Lothian, UK \\ ${ }^{4}$ Centre for Population Health Sciences, General Practice Section, University of Edinburgh, Edinburgh, UK \\ ${ }^{5}$ Department of Medical Sciences, University of Edinburgh, Edinburgh, UK
}

\begin{abstract}
Aim: To establish the acceptability of telemetric monitoring of blood pressure to patients and clinicians. Background: Telemetric monitoring of blood pressure (BP) may allow clinicians and patients, in partnership, to more quickly control high BP through medication and lifestyle alterations. However, it is not clear if patients and clinicians would find such a system acceptable. Methods: Questionnaire study followed by focus groups of patients with high BP, and clinicians involved in managing BP. Findings: We received responses from $25(50 \%)$ practice nurses, $76(50 \%)$ general practitioners and $126(62 \%)$ patients. We ran three focus groups of patients and clinicians. Participants were supportive of the technology, willing to try it, thought it would encourage adherence to medication and lifestyle and felt it would diagnose problems sooner than current methods. However, both groups thought the technology would be more useful for new patients or those whose BP was uncontrolled. They were concerned that individual high readings might provoke anxiety and thought patients would need reassurances about this. Clinicians were concerned about workload and the responsibility to act immediately when faced with a continuous stream of readings, regardless of how inconvenient this may be. Conclusion: Participants, in general, welcomed this technology and thought it would aid adherence to medication and lifestyle advice, but felt it was most suitable to those with newly diagnosed or uncontrolled hypertension. Patients will need to be educated and reassured about the nature of occasional high readings and the need to consider average BP.
\end{abstract}

Key words: hypertension; qualitative research; telemedicine

Received 21 January 2009; accepted 7 June 2009; first published online 28 July 2009

\section{Introduction}

Intervention trials show that every $10 \mathrm{mmHg}$ fall in systolic blood pressure (BP) is associated with

Correspondence to: Brian McKinstry, Reader in Primary Care Research, Centre for Population Health Sciences, General Practice Section, 20 W Richmond Street, Edinburgh, EH8 9DR, UK. Email: brian.mckinstry@ed.ac.uk

(C) Cambridge University Press 2009 reduction in risk of stroke and coronary events of $40 \%$ and $20 \%$, respectively (Poulter et al., 2005). The time taken to achieve control also has a significant impact on event rates (Julius et al., 2004). Despite this, BP control in practice remains inadequate, with slow responses to high surgerybased measurements (Primatesta and Poulter, 2004). Self-monitored BP predicts outcome as 


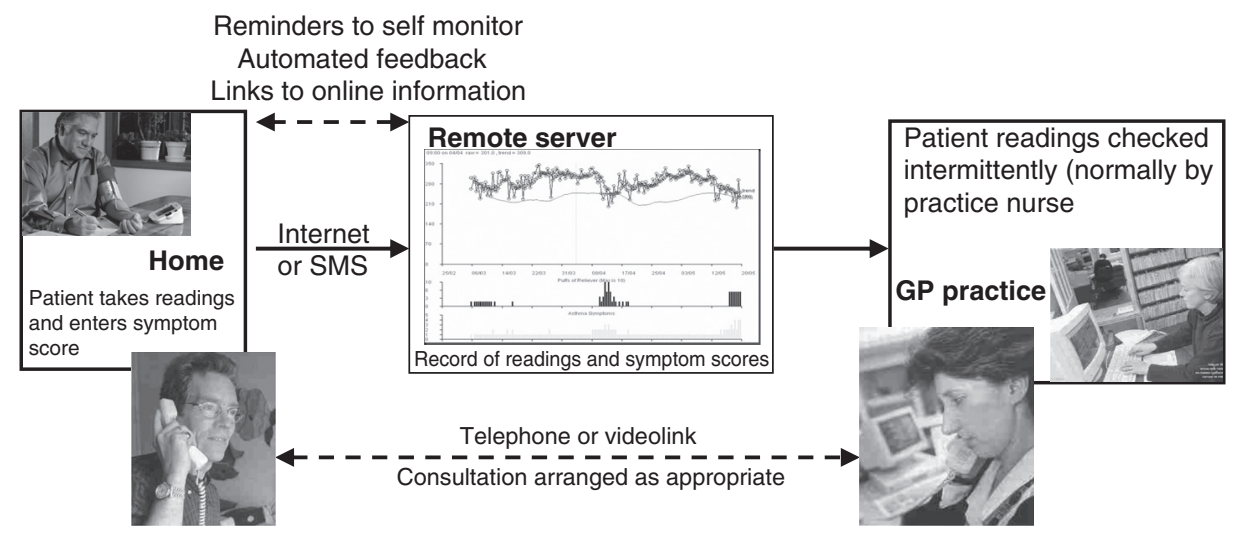

Figure 1 Models of telemetric supported self-monitoring

well as ambulatory measurement (the 'gold standard') (Stergiou et al., 2004) and both better than surgery-based measurements. However, there is conflicting evidence on whether unsupervised home monitoring improves outcomes (Schroeder et al., 2004) and it is known that in type 2 diabetes it may actually be harmful (Farmer et al., 2007). Evidence from qualitative research has suggested that, in part, this is due to patients feeling that they have insufficient information to deal with abnormal results themselves and feel bereft of clinician support (Peel et al., 2007).

Telemetry and mobile phone-based technologies are now available which have the potential to change the dynamics of monitoring and selfmanagement by enabling patients to monitor their condition, while allowing clinicians to continue to take an overview (see Figure 1). In addition, intelligent software advice programmes, can give personalized feedback on appropriate action at different BP levels. Information is simultaneously forwarded to a clinician through Internet connections, which can highlight patients whose control is inadequate, thus facilitating timely contact.

Telemetric systems have been shown to improve compliance with intensive insulin regimes in teenagers (Franklin et al., 2006), reduce hospital admissions in people with heart failure (Louis et al., 2003), increase treatment adherence in hypertension (Friedman et al., 1996), and to improve monitoring and increase self-management confidence among people with asthma (Pinnock et al., 2007). Limited data on telemetry-supported home BP monitoring from other countries suggests it can improve BP control (Rogers et al., 2001; Artinian et al., 2007; Parati et al., 2009).

Whilst prescribed drugs are seen as the mainstay of therapy, patient involvement in management is critical in terms of concordance with therapy and important lifestyle choices such as weight control, exercise, and salt and alcohol intake (North of England Hypertension Guideline Development Group, 2004). We found, in our recent randomized controlled trial, that providing information alone is ineffective in reducing $\mathrm{BP}$ (McKinstry et al., 2006). Unlike conditions such as asthma, hypertension is nearly always asymptomatic and hitherto patients have not been able to get direct feedback on how well they are doing in controlling their condition. Telemetric monitoring and feedback, therefore, have the potential to influence lifestyle adherence.

The importance of user involvement in the design of technological interventions is well recognized, not only to establish a target audience for whom it is likely to be successful, but also to ensure that the technical solution meets the needs of an organization and avoids unintended consequences on working practices (Dillon, 2001; May et al., 2007). Before planning a randomized controlled trial using this mobile phone-based monitoring (e-BP), we wanted to establish any concerns patients and clinicians may have about this type of technology, particularly with regard to likely ease of use, confidentiality, appropriateness 
of warnings, frequency of monitoring, impact on workload, potential of provoking anxiety and the types of patients and scenarios in which the technology would be best employed. We also wished to see how commonly patients and clinicians made use of home-monitoring results in managing their BP.

\section{Methods}

The study was conducted in Lothian, Scotland, in the summer of 2007. We employed a mixed methods approach, using questionnaires and focus groups to gather the views of general practitioners (GPs), practice nurses (PNs) and patients, on self-monitoring of high BP. Ethical approval was obtained from the Lothian Research Ethics Committee.

\section{Questionnaire}

Self-administered questionnaires were designed for clinicians and patients, based on those used successfully in our survey of patients with asthma (Pinnock et al., 2006) and derived from literature on the acceptance of technology (Moore, 1991; Rogers, 2003). The relative importance of potential issues was assessed by asking respondents to rate their agreement with statements on a 5-point Likert scale $(1=$ strongly agree; $5=$ strongly disagree), or to choose between two opposing positions on a scale of 1 to 10 . The option of adding comments in free text was also provided. Minor adjustments were made after piloting with clinician colleagues and patients.

Prior to completing the questionnaire, clinicians and patients were given a written description of how an e-BP measuring system might work. In the system, described patients are texted or e-mailed a message which explains if their BP is controlled or uncontrolled and advises either to continue with medication and lifestyle advice or to contact their clinician, as appropriate. The clinician can also access their patients' readings and are expected to do so about once a week (see Figure 1).

\section{The clinician questionnaire}

The clinician questionnaire sent used both closed and open questions and asked about general attitudes toward using technology and specifically about the potential value of a patient-monitored clinician-supervised e-BP service. Opinions were sought on relevance to general practice, practicability and clinical situations in which monitoring using mobile technology might be helpful.

\section{The patient questionnaire}

This was designed to ascertain patients' attitudes toward technology in general and opinions on the likely effect on their BP control, including adherence to drug therapy and lifestyle advice, convenience and clinical situations in which such technology might be helpful.

\section{Sample}

A sample of 150 NHS GPs and 50 PNs in Lothian was selected from 50 practices across Lothian. Four practices, chosen to reflect a range of socio-economic conditions, were recruited. A random sample of a total of 200 adults, from the practices' hypertension registers, stratified by age and sex, was selected. One reminder was sent to non-respondents after an interval of four weeks. In addition to requesting completion of the questionnaire, participants were asked if they would be willing to participate in the qualitative stage of the research.

\section{Analysis}

Data were entered into SPSS 11 (Statistical Package for the Social Sciences, 2008). We performed a descriptive analysis and where appropriate, analyses were undertaken to explore the relationships between age/demographic profiles and attitudes to mobile technology using $t$-tests.

The free text responses to open-ended questions were coded and emerging themes identified, and used to inform the topic guides for the focus groups.

\section{Focus groups}

Focus groups have the advantage of making use of group dynamics to stimulate discussion, yield insights, generate ideas and to pursue a topic indepth (Strauss and Corbin, 1998), allowing participants to discuss each others' perspectives.

\section{Sample}

We used responses to the postal questionnaire to select clinicians and patients representing a 
range of age groups, gender, practice demography and attitudes to the use of mobile technology.

\section{Format of the focus groups and data collection}

We opted to run separate groups for clinicians and patients in order to encourage sharing of experiences in a 'safe' environment, uninfluenced by professional power (Strauss and Corbin, 1998). The focus groups were held in convenient locations (health or community centres). Outline topic guides were developed from the literature and following multi-disciplinary discussion, which included representatives from academia, practicing GPs (some with a special interest in BP care) and experts in informatics. Further refinement incorporated themes emerging from the free text responses of the clinician and patient questionnaires.

Focus groups started with a description and demonstration of e-BP monitoring technology, which prompted questions and stimulated discussion.

Focus group discussions were facilitated by the study researcher, with at least one other researcher present. They were audio-taped, with consent, and comprehensive field notes taken. The hour-long discussions were then transcribed verbatim and a thematic analysis conducted. Transcripts were independently reviewed by another researcher to be sure that no important themes had been overlooked.

\section{Results}

\section{Questionnaire}

From 50 PNs, 150 GPs and 200 patients, we had $25(50 \%), 76(50 \%)$ and $126(62 \%)$ responses, respectively. The sample reflected a range of clinicians and patients of different ages, rurality, socio-economic groups, and different sizes and types of practice. In all, $95 \%$ of the nurses, $47 \%$ of the GPs and $50 \%$ of the patients responding were female, largely reflecting Lothian demographics (see Table 1).

\section{Responses of GPs and nurses to the questionnaire}

\section{Attitudes to technology}

On a 10-point scale rating attitude to four statements highlighting general attitudes toward
Table 1 Demography of respondents to questionnaire

\begin{tabular}{lccc}
\hline Age & $\begin{array}{l}\text { Nurses } \\
n=26(\%)\end{array}$ & $\begin{array}{l}\text { GPs } n=76 \\
(\%)\end{array}$ & $\begin{array}{l}\text { Patients } \\
n=126(\%)\end{array}$ \\
\hline $23-29$ & 4 & 0 & 0 \\
$30-39$ & 16 & 21 & 2 \\
$40-49$ & 56 & 42 & 19 \\
$50-59$ & 24 & 36 & 28 \\
$60-69$ & 0 & 1 & 24 \\
$70-75$ & 0 & 0 & 8 \\
Over 75 & 0 & 0 & 12 \\
Not stated & 0 & 0 & 9 \\
\hline
\end{tabular}

$\mathrm{GPs}=$ general practitioners

new technologies, GPs and nurses demonstrated a willingness to try out new systems, were generally confident about learning to use and using new equipment and saw modern technology as an important part of their lives, with a modal score of 7 or 8 for most questions (6 or above being positive).

\section{Use of home monitors}

In all, $60 \%$ of nurses and $34 \%$ of GPs routinely lend home monitors to patients to measure their BP. They do this mainly for diagnostic reasons $(80 \%)$ and to measure BP if it is found to be high, but less frequently to monitor alterations in treatment or routine measurement. Only a minority of GPs $(25 \%)$ and nurses $(40 \%)$ routinely asked patients if they had home monitors. Although a small minority (16\% of nurses and $9 \%$ of GPs) would never encourage patients to self-monitor, most clinicians $(69 \%)$ occasionally encouraged patients to use home monitoring and would ask patients to bring their home records to the surgery. A total of $36 \%$ of nurses and $20 \%$ of GPs checked patients' home devices for accuracy against surgery devices.

\section{Ambulatory blood pressure measurement}

One third of the clinicians indicated that their practice had one or more ambulatory blood pressure monitors (ABPM), and $52 \%$ of nurses and $82 \%$ of GPs said they had easy access to ABPM in secondary care. Doctors and nurses used the devices mainly for diagnosis and for 'difficult to control' hypertensives. Only $8 \%$ of clinicians used them routinely in all new hypertensives. 
Attitudes to mobile telephone technologies in blood pressure monitoring

The responses to the statements about practical applicability of e-BP monitoring, showed that, overall, the attitude towards this new technology was positive, with $64 \%$ of nurses and $63 \%$ of GPs agreeing strongly or very strongly that it would aid them in their job and also help patients to follow their recommendations to monitor their readings and take medication. Altogether, $80 \%$ of clinicians agreed strongly or very strongly that they would wish to be alerted if their patient had a very high reading (systolic $>200$ ) and $60 \%$ agreed that it would be useful to have access to all the patients' readings. Nurses (84\%) and GPs $(74 \%)$ showed an interest in trying out this technology. A total of $70 \%$ of clinicians thought that reminder texts to patients would improve regular measurement of BP. Most did not indicate concerns over patient confidentiality or that the technology might induce dependence.

\section{Patient responses}

Home BP monitor, mobile phone and Internet use

In total, $31 \%$ of patients who responded had a home monitor. Of those who had one, $47 \%$ checked their BP more than once a week and an additional 16\% more than once a month. Among the respondents, $84 \%$ of men and $70 \%$ of women had a mobile phone, of whom $55 \%$ used text, but only $3 \%$ accessed the Internet on it despite most having phones with the ability to do so. Internet access was available to $69 \%$ of respondents.

\section{Attitudes to technology}

Patients were more diverse in their views on their attitudes to technology than clinicians. Although $23 \%$ of patients were inclined to the view (score of 4 or less on a 10 point scale) that 'technology was not an important part of their lives', $41 \%$ felt that they 'could not imagine life without modern technology' (score of 7 or more in a 10 point scale). In general, male patients were more likely to have favourable attitudes to the technology than female patients, as were those below 60 years compared with those above 60 years of age (see Table 2).

In all, $51 \%$ of patients agreed or strongly agreed that the e-BP technology would help them

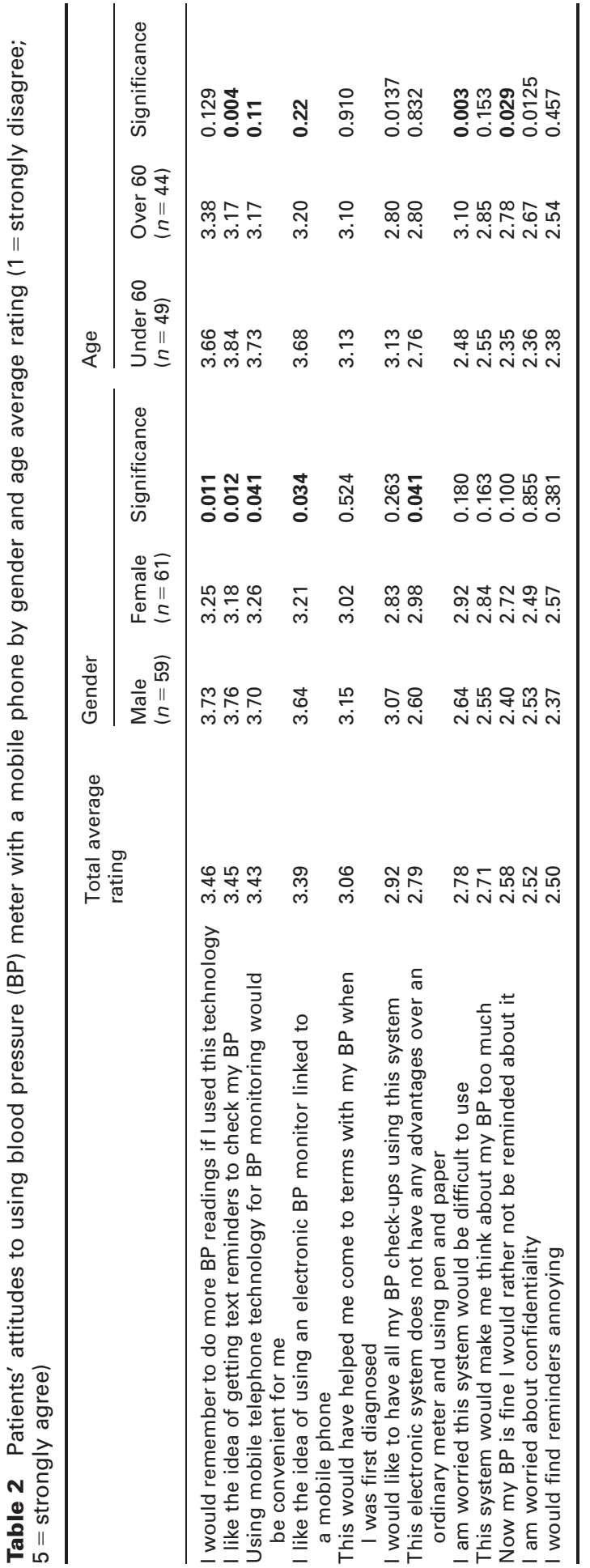

Primary Health Care Research \& Development 2009; 10: 299-308 
remember to check their BP and liked the idea of using the system. Attitudes to receiving texts reminding them to check their BP were mixed, with $50 \%$ agreeing that they 'liked the idea', but $23 \%$ disagreed or strongly disagreed with this idea. A quarter thought the system might be difficult to use and a similar number were concerned that it might make them think of their BP too much. Few (12\%) were worried about confidentiality. Most (64\%) felt that the technology would help their clinicians manage their BP.

\section{Qualitative study}

Two patient and one clinician focus groups were undertaken involving 25 people in total (see Table 3).

The general response of both patients and clinicians to e-BP monitoring was generally positive, even from those patients who did not own mobile phones. PNs were most enthusiastic.

This would be great for me - it sounds too good to be true! There is a lot of routine where we don't need to see someone face-to-face

$(\mathrm{PN})$

\section{Potential benefits}

\section{Improved access}

Patients appreciated the potential for e-BP systems to reduce the need for routine visits to the surgery which, for a number of participants, were difficult. A specific group highlighted by the clinicians was pregnant women with pre-eclampsia who may otherwise require daily visits from a midwife.

Improved diagnosis, close monitoring and early identification of problems

Tele-monitoring would pick up if BP was changing, which would mean that intervention could happen sooner rather than later. One patient related his experience to general agreement within the group that e-BP monitoring could facilitate regular monitoring.

This system would be better. I had a problem about two months ago - I missed my sixmonth one because I'm bad about going back in and I was called back in because
Table 3 Focus group participants

\begin{tabular}{llll}
\hline & \multicolumn{2}{l}{ Age } & \\
\cline { 2 - 4 } & $23-45$ & $46-65$ & $>65$ \\
\hline $\begin{array}{l}\text { Health professionals } \\
\quad \text { Nurses }\end{array}$ & 1 & 2 & - \\
$\quad$ GPs & 3 & 3 & - \\
Patients & & & \\
$\quad$ Male & & 4 & 2 \\
Female & 4 & 3 & 1 \\
\hline
\end{tabular}

$\mathrm{GPs}=$ general practitioners.

I was quite unwell. This would have been an advantage as it would encourage me to check it more regularly - I don't know how often I would have to check it with this system, once a day or week? - and my high BP would have been pointed out to me earlier.

(Female patient participant)

Patients also felt that it would be useful in new diagnoses and borderline cases. One participant felt that it would have been particularly beneficial to establish an average reading:

I think it would have benefited me a lot to be honest, and I think I probably missed out because my treatment was quite erratic at the start and I was put on treatment for about three months and then taken off it because it came down but then it went away back up again and I don't know when it went away back up - it's just I started feeling unwell and that's why I went back about six/seven months later and it was sky high again.

(Male patient participant)

In contrast, those patients who felt that their BP was well managed found it difficult to understand what might be the benefits to them.

I don't really get this. What's the point if you're going to the doctor every four months or so and I take my medication every day then what's the point in this.

(Female patient participant)

Clinicians agreed that certain groups of patients would be more likely to benefit, for example those who either needed close monitoring (eg, post-stroke or diabetic patients) or newly diagnosed patients where doses of drugs were being titrated. 
Empowering self-care

The potential to encourage and facilitate selfcare was mentioned by a number of patients and professionals. These included reminding people to make lifestyle changes that would help reduce their BP and take more notice of their BP than they currently do. Patients also felt that continuous monitoring would have the effect of ensuring that they took their medication.

Yes I would do something about it for example if it was too high. ... . I think if I've been doing anything differently or is there anything I can do? If it was getting higher I would be thinking I definitely need to do something about it.

(Male patient participant)

The system was also seen as putting patients more in control, but with both patient and GP sharing responsibility:

...... if the people looking after you think you're having a problem, they can do something straight away. Instant communication is good. At the moment I rely on my GP and my health centre, they write me a letter and I go in, they check my BP and that's it until the next letter or phone call.

(Male patient participant)

I have a spreadsheet where I keep all my results and I can work out my average. I just got a printout of all my results and took it along to the doctor and it meant that when he was deciding what to give me I could record what medication I had at what point and so he could see the average and the effect of the medication. It was really useful from that point of view but I think this (telemetry system) will make it a lot easier as there's less to do.

(Male patient participant)

\section{Potential disadvantages}

Increasing anxiety

The main concern expressed was that continuous monitoring of BP might increase patients' anxiety over their condition. Currently, between visits for BP checks, apart from taking tablets, many could forget about their BP, and some expressed concern that frequent monitoring would be very worrying.

I would be quite worried if I took it all the time and it was high. I just think I'm perfectly all right until I go to the surgery in four months time. I don't worry about it. I don't think about it. Although I take my tablets every day but I think if I did all that I'd be a wee bit anxious.

(Female patient participant.)

Mindful of the natural variability of BP, clinicians were concerned about the nature of any 'warning messages' that their patients would receive via the text-messaging system after submitting an unusually high BP reading, and were concerned that feedback might worry patients unnecessarily. Patients varied and might require different messages. For example, there was general agreement that the system would be less suitable for more anxious patients.

I would be concerned that all these patients would be getting messages that their BP is up and worrying about it all weekend.

(GP)

Workload, responsibility and cost-effectiveness

A key concern was whether or not the technology would increase workload for clinicians. They were also worried about responsibility, observing that once a clinician had this information they would be duty-bound to act on it within a reasonable time frame.

Once you have the information you become responsible. It would be $\mathrm{OK}$ to look at it once a week - I would worry what to do if I had the information at $5.30[\mathrm{pm}]$.

Some clinicians were concerned about the cost of the telemetry equipment, particularly as they felt that the scope for improving $\mathrm{BP}$ control was limited due to patients already being on maximally tolerated drug regimes. Some clinicians were also concerned about the ability of patients to use the equipment. Those who loaned monitors to patients wondered to what extent the e-BP would add benefit.

I can't see any benefits of this over what we already do. And I can't see us using this 
system any differently to the way we use the ones we give patients to take away.

Views on messaging

Participants were asked their views about receiving advisory text messages. The groups had three potential messages suggested to them: BP controlled and advice to continue therapy and lifestyle measures; average BP high and advised to make a routine contact with their nurse or doctor; and, if the BP was very high, a message to contact the surgery urgently.

There was no clear consensus as to the best wording for the messages. Participants felt that some may react badly to an urgent message to contact the surgery and panic. Clear explanations about the meaning of averages and the implication of the short messages for the individual patient would be as important as the precise wording used. Information should include explanations of the readings, range, variation and averages, as well as defining the target for the individual. Some felt that a phone call from the GP or nurse would be preferable to a text message, when average BP was high. Patients wanted to have a clear understanding of how their doctor would access and use the information, including how often they would access the information.

One group suggested using a traffic light system rather than verbal text messages, (with green = 'fine', amber = 'watch it' and red = 'see your doctor') although again there was some concern as to how patients might react should they receive a red alert.

\section{Acceptability}

The view was expressed by both clinicians and patients that increased availability of home monitors is such that more patients are familiar with using them and therefore the introduction of such a system would be relatively easy with some groups. There were, however, reservations about the ability of older groups to use the technology. Clinicians expressed some concerns about the security of data and reassurances were sought on this issue.

\section{Discussion}

This was a small study in one region of the UK and, therefore, must be interpreted with caution.
In particular, it is likely that more interested professionals and patients will have chosen to take part. However, response rates were reasonable for postal surveys and participants represented a wide range of ages, genders and attitudes to technology. We did not undertake full psychometric testing of our questionnaire, however, it was based on questions previously used successfully in a similar project exploring attitudes to technology in asthma and had good face validity when tested at the pilot stage. We were aware that the researcher's positive attitude to the concept of tele-health might affect data generation and interpretation (Bowling, 2002) and so we aimed to foster an environment that allowed participants to express both positive and negative attitudes towards the e-BP technology, if they wished. In addition, we adopted a multi-disciplinary team approach throughout the study (Barrie et al., 1999) to ensure that a range of perspectives was reflected in the final interpretation.

Both professional and patient participants were enthusiastic about the use of tele-monitoring to manage BP, and most willing to try out the systems. Even allowing for the fact that our respondents were probably more interested, the results suggest that a sizeable proportion of the hypertensive population would be willing to try tele-monitoring. This reflects the findings in other studies of chronic conditions in which such interventions have been well received by patients (Friedman et al., 1996; Louis et al., 2003; Franklin et al., 2006).

It is clear from both professionals and patients that this technology has particular role in the assessment and management of newly diagnosed patients, those who are uncontrolled or in special circumstances such as pre-eclampsia and that it has less to offer 'controlled' patients with monitoring being scaled down once this had been achieved. This fits with the theoretical model developed by Glasziou et al. (2005), who describe the complementary and evolving roles of periodic professional reviews and ongoing patient selfmonitoring. A newly diagnosed condition is assessed and brought under control with professional support, before the patient assumes responsibility for self-management as the stable maintenance phase is established.

Given the concerns around engendering anxiety expressed in our study, it will clearly be 
important, when enroling patients to a telemonitoring service, to explain the meaning of individual and average $\mathrm{BP}$ readings and that a raised $\mathrm{BP}$ reading does not constitute an immediate risk to them. Our previous work with asthmatic patients (Pinnock et al., 2007), suggested that although tele-monitoring had the potential to facilitate self-care, there was a paradoxical risk of increasing dependence on professional support. In general, however, our participants perceived the process as one which encouraged self-care rather than induced dependency.

It will be interesting in subsequent trials to see if the perceived benefits on lifestyle and medication adherence actually accrue and if concerns about the impact on anxiety are realized.

\section{Conclusion}

This study revealed enthusiasm among participating professionals and patients for trying out a system of telemetric monitoring for high BP. However, both patients and practitioners thought that, at least initially, it was likely to be more useful to new patients and those whose BP was above the target level or who needed intensive monitoring. When introducing such a system, the nature of the feedback messages and the supporting education about implication of single high readings will be crucial.

\section{Acknowledgements}

We would like to thank all the practices, patients, doctors and nurses who took part, and Kelly McGorm of the Scottish Primary Care Research Network who helped with recruitment. This study was funded by the Royal College of General Practitioners' Scientific Foundation. BM and HP are supported by fellowships from the Chief Scientist Office of the Scottish Government.

\section{References}

Artinian, N., Flack, J., Nordstom, C., Hockman, E., Washington, O., Jen, K.-L. et al. 2007: Effects of nursemanaged telemonitoring on blood pressure at 12-month follow-up among urban African Americans. Nursing Research 56, 312-22.
Barrie, C., Britten, N., Barber, N. and Bradley, S. 1999: Using reflexivity to optimise teamwork in qualitative research. Qualitative Health Research 9, 26-44.

Bowling, A. 2002: Research methods in health: investigating health and health services. Buckingham: Open University Press.

Dillon, A. 2001: User acceptance of information technology. In Karwowski, W., editor, Encyclopedia of human factors and ergonomics. London: Taylor and Francis.

Farmer, A., Wade, A., Goyder, E., Yudkin, P., French, D., Craven, A., Holman, R., Kinmonth, A.-L. and Neil, A. 2007: Impact of self-monitoring of blood glucose in the management of patients with non-insulin treated diabetes: open parallel group randomised trial. British Medical Journal 335, 132-36.

Franklin, V., Waller, A., Pagliari, C. and Greene, S. 2006: Randomised controlled trial of 'Sweet Talk', a text messaging system to support young people with diabetes. Diabetic Medicine 23, 1332-38.

Friedman, R., Kaziz, L., Jette, A., Smith, A., Stollerman, J., Torgerson, J. and Carey, K. 1996: A telecommunications system for monitoring and counseling patients with hypertension: impact on medication adherence and blood pressure control. American Journal of Hypertension 9, 285-92.

Glasziou, P., Irwig, L. and Mant, D. 2005: Monitoring in chronic disease a rational approach. British Medical Journal 330, 644-48.

Julius, S., Kjeldsen, S., Weber, M., Brunner, H., Ekman, S., Hansson, L., Hua, T., Laragh, J., McInness, G., Mitchell, L. et al. and VALUE trial group 2004: Outcomes in hypertensive patients at high cardiovascular risk treated with regimens based on valsartan or amlodipine: the VALUE randomised trial. Lancet 363, 2022-29.

Louis, A., Turner, T., Gretton, M., Bakosh, A. and Cleland, J. 2003: A systematic review of telemonitoring for the management of heart failure. European Journal of Heart Failure 5, 583-90.

May, C., Finch, T., Mair, F., Ballini, L., Dowrick, C., Eccles, M., Gask, L., MacFarlane, A., Murray, E., Rapley, T., Rogers, A., Treweek, S., Wallace, P., Anderson, G., Burns, J. and Heaven, B. 2007: Understanding the implementation of complex interventions in health care: the Normalization Process Model. BMC Health Services Research 7, 148.

McKinstry, B., Hanley, J., Heaney, D., McCloughan, L., Elton, R. and Webb, D. 2006: Impact on hypertension control of a patient-held guideline: a randomised controlled trial. British Journal of General Practice 56, 842-47.

Moore, G. 1991: Crossing the chasm. New York: HarperBusiness.

North of England Hypertension Guideline Development Group. 2004: Essential hypertension: managing adult patients in primary care. Newcastle: Centre for Health Services Research, University of Newcastle, 111.

Parati, G., Ombonid, S., Albinia, F., Piantonia, L., Giulianoa, A., Revera, M., Illyese, M. and Mancia, G.; on behalf of the 
TeleBPCare Study Group 2009: Home blood pressure telemonitoring improves hypertension control in general practice. The TeleBPCare study. Journal of Hypertension 27, 198-203.

Peel, E., Douglas, M. and Lawton, J. 2007: Self monitoring of blood glucose in type 2 diabetes: longitudinal qualitative study of patients' perspectives. British Medical Journal 335, 493-97.

Pinnock, H., Slack, R., Pagliari, C., Price, D. and Sheikh, A. 2006: Professional and patient attitudes to using mobile phone technology to monitor asthma: questionnaire survey. Primary Care Respiratory Journal 5, 583-90.

Pinnock, H., Slack, R., Pagliari, C., Price, D. and Sheikh, A. 2007: Understanding the potential role of mobile phonebased monitoring on asthma self-management: qualitative study. Clinical and Experimental Allergy 37, 794-802.

Poulter, N., Wedel, H., Dahlof, B., Sever, P., Beevers, D., Caulfiel, M., Kjeldsen, S., Kristinsson, A., McInnes, G. et al. 2005: Role of blood pressure and other variables in the differential cardiovascular event rates noted in the AngloScandinavian Cardiac Outcomes Trial-Blood Pressure Lowering Arm (ASCOT-BPLA). Lancet 366, 907-13.

Primatesta, P. and Poulter, N. 2004: Hypertension management and control among English adults aged 65 years and older in 2000 and 2001. Journal of Hypertension 22, 1093-98.

Rogers, E. 2003: Diffusion of innovations. New York: Simon \& Schuster.

Rogers, M., Small, D., Buchan, D., Butch, C., Stewart, C., Krenzer, B. et al. 2001: Home monitoring service improves mean arterial pressure in patients with essential hypertension: a randomized, controlled trial. Annals of Internal Medicine 134, 1024-32.

Schroeder, K., Fahey, T. and Ebrahim, S. 2004: Interventions for improving adherence to treatment in patients with high blood pressure in ambulatory settings. Cochrane Database of Systematic Reviews no. 3, CD004804, doi:10.1002/ 14651858.CD004804.

Statistical Package for the Social Sciences. 2008: Chicago, SPSS.

Stergiou, G., Mengden, T., Padfield, P., Parati, G. and O'Brien, E. 2004: Working group on blood pressure monitoring of the European Society of Hypertension. Self monitoring of blood pressure at home. British Medical Journal 329, 870-71.

Strauss, A. and Corbin, J. 1998: Basics of qualitative research, second edition. London: Sage. 